\title{
Role of Ki 67 Labelling Index as an Adjunct to Histopathological Diagnosis for Grading of CNS Tumours
}

\author{
Priyanka Rai ${ }^{1}$, Chandni Krishnani², S. S. Goswami ${ }^{3}$ \\ ${ }^{1}$ Department of Pathology, SBKSMI and RC, Dr. Lal Path Labs, Varanasi, Uttar Pradesh, \\ India. ${ }^{2}$ Department of Pathology, SBKSMI and RC, RIMS, Raipur, Chhattisgarh, India. \\ ${ }^{3}$ Department of Pathology, SBKS Shah and RI, Vadodara, Gujarat, India.
}

\section{ABSTRACT}

\section{BACKGROUND}

The Central Nervous System tumours are unique as they arise from specialized tissue. CNS tumours constitute a wide range of neoplasm that differs in their location, age distribution, extent of invasiveness, morphological features and tendency for progression. We wanted to evaluate the traditional morphological data with knowledge on the prognostication marker Ki 67 antibody in evaluating tumour grade and prognosis of CNS neoplasm.

\section{METHODS}

This is a cross section study carried out between March 2015 and September 2016 in histopathology department of Dhiraj Hospital on 50 cases of CNS tumours. Immunolabelling of all biopsies was done by horse radish peroxidase technique using rabbit monoclonal antibody to Ki 67 (clone SP 6) (Thermo Scientific, USA). Ki 67 immune positive labelling index was obtained for each tumour and correlated with mitotic labelling index obtained by conventional morphological grading as per WHO 2007 classification.

\section{RESULTS}

In our study of CNS tumours, all age groups were studied. The mean Ki 67 labelling index (LI) values +/-SD for WHO Grade I tumours were- meningiomas (10) 3.85 $(+/ 1.97) \%$, schwannoma (3) -3.0 (+/-2.97) \%, pituitary adenoma (1) 0.6, craniopharyngioma (1) $-1.1 \%$ and ependymoma (6) $2.62(+/-0.60) \%$. WHO Grade II tumours- atypical angiomatous meningioma (1) $-2 \%$, atypical mucinous meningioma (2) $-6.15(+/-1.06) \%$, gemistocytic astrocytoma (1) -12; pleomorphic xanthoastrocytoma (2) $-4.3(+/-0.99) \%$, astrocytoma grade ii (2) $-3.3(+/-0.71) \%$, oligodendroglioma grade ii (4) - $3.9(+/-0.88) \%$. WHO grade III tumours- anaplastic astrocytomas (5) $-6.82(+/-2.17) \%$, anaplastic oligoastrocytoma grade iii (1) -10.8 $\%$. who grade iv-glioblastomas (7) -18.44 (+/-3.97) \%; medulloblastomas (1) 20\%, metastatic tumour (3) $-36(+/-22.16) \%$. In our study, the mean Ki 67 LI $( \pm$ SD) values for grade II, III and IV glioma is as follows: $4.76(+/-2.83) \%, 7.48(+/-2.53) \%$ and 18.44 (3.97) \% respectively.

\section{CONCLUSIONS}

This study shows that Ki 67 LI serves as an essential clinical prognostic proliferation marker of particular importance in cases with lower grade histology of Grade II \& Grade III astrocytomas, Grade II \& Grade III oligodendrogliomas. Ki 67 LI is important in determining benign, atypical and malignant meningiomas, noninvasive and invasive pituitary adenomas.

\section{KEY WORDS}

CNS, Brain, Spinal Cord, Tumours, Immuno-Histochemistry, Ki 67/MIB-1
Corresponding Author: Dr. Chandni Krishnani, A5, Capital City, Phase III, Saddu, Raipur-492014, Chhattisgarh, India. E-mail: itschandnik@gmail.com

DOI: $10.14260 / \mathrm{jemds} / 2020 / 290$

Financial or Other Competing Interests: None.

How to Cite This Article:

Rai P, Krishnani C, Goswami SS. Role of Ki 67 labelling index as an adjunct to histopathological diagnosis for grading of CNS tumours. J. Evolution Med. Dent. Sci. 2020;9(16):1331-1335, 10.14260/jemds/2020/290

Submission 30-01-2020, Peer Review 25-03-2020, Acceptance 03-04-2020, Published 20-04-2020. DOI:

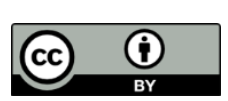




\section{BACKGROUND}

The central nervous system tumours are unique as they arise from specialized tissue. CNS tumours constitute a wide range of neoplasm that differs in their location, age distribution, extent of invasiveness, morphological features and tendency for progression.

CNS tumours may be

a. Primary intracranial tumours, which arise from brain or pituitary gland or their coverings

b. Secondary intracranial tumours which are due to local extension of regional tumour or metastatic primary malignancy from elsewhere body. ${ }^{1}$

According to WHO classification of CNS tumours, CNS tumours are grouped into four grades (1 to IV) on the basis of "cellularity, nuclear atypia, mitotic activity, pseudo palisading necrosis and or microvascular proliferation". A strong correlation has been observed between WHO histological grade and patient survival.(2) Measurement of proliferative activity of CNS tumour is important in determining the tumour grade, recurrence rate and malignancy.(3) Measurement of cell proliferation in tumour tissue can be performed using "Bromodeoxyuridine Labelling Index (BrdU LI), flow cytometry (FCM), MIB-1 antibody to the Ki 67 antigen (MIB-1), proliferating cell nuclear antigen (PCNA), and argyrophilic nucleolar organising regions (AgNOR)". At the present time Ki 67/MIB-1 and AgNOR are the simplest and most reliable of techniques.(4)

In studies on Ki 67 expression among brain tumours, higher Labelling index were associated with histological malignant neoplasm including metastatic carcinomas.(5) A higher Ki-67 LI was associated with higher histological grade and poorer survival.(6) Studies have suggested that the Ki 67 LI is more significantly related to the survival than the modified Ringertz histopathology grading system of astrocytic gliomas.(6) The mean Ki 67 LI of low grade astrocytoma is shown to differ significantly from both anaplastic astrocytoma and glioblastoma.(6) MIB-1 is expected to be useful in cases where clinical or histopathological factors are equivocal or where they cannot be fully assessed (7) Multivariate analysis have also shown that MIB-1 generally has excellent sensitivity and specificity in placing patients correctly into groups of survivors and non survivors.(8)

We wanted to study of Ki 67 Labelling Index in CNS tumours, classify and Grade CNS tumours according to WHO classification, determine $\mathrm{Ki} 67$ Labelling Index of CNS tumours and test the differences statistically of Ki 67 labelling index with mitotic index and with grade of CNS tumours.

\section{METHODS}

This study was cross sectional study all CNS tumour specimens sent to Dhiraj hospital. Conventional histomorphologically graded (according to WHO 2007 classification) ${ }^{(9)}$ tumours were reviewed for mitotic labelling which were subjected to immunolabelling by proliferative marker Ki 67 antibody to obtain Ki 67 LI.(10,11,12,13) The study includes all age groups operated for CNS neoplasm and satisfying inclusion criteria. Sample size of subjects was 50 cases of CNS tumours surgically removed and submitted for histological examination immediately fixed in $10 \%$ buffered formalin to Dhiraj hospital during the study period. The sample size was taken based on the convenience of the study. One year study carried out from March 2015 to September 2016 in the Histopathology section of Pathology department equipped with immuno-histochemistry. The study was approved by IEC and informed consent was obtained. The study was carried on routine paraffin sections or blocks after antigen retrieval using KI-67/MIB1 antibodies along with controls. Light microscopy was used for reading of slides. The regions with highest concentrations of MIB-1 positive nuclei was selected and analysed at high power magnification. On the basis of at least 1,000 neoplastic nuclei, the KI-67 labelling index was calculated in each slide as the percentage of immune positive nuclei. Vascular components and haematogenous cells were excluded. Only the dark brown stained nuclei were considered as immune positive.

\section{Inclusion Criteria}

All age group patient post-operative biopsies, Metastatic CNS neoplasm, recurrent neoplastic CNS lesions and Stereotactic biopsies sent to the laboratory for CNS neoplasm.

\section{Exclusion Criteria}

a. Non neoplastic CNS lesions.

b. Improper fixative/unfixed tissues where antigen retrieval is not possible.

c. Non representative CNS tissues (tumour not identifiable in paraffin block).

\section{Ki 67 Labelling Index- Procedure}

Immunohistochemical staining by the Horse radish peroxidase technique using rabbit monoclonal antibody to Ki 67 [clone SP 6] (thermo scientific, USA) was performed.(14,15) The slides are developed using freshly prepared diaminobenzidine tetra hydrochloride solution (DAKO Envision FLEX DAB + chromogen) Mitotic count was counted as number of mitoses in ten consecutive HPFs and one of the grading criteria of WHO classification 2007. Mitotic labelling index was calculated as, the percentage of mitoses per 1000 cells on H\&E sections. The "proliferation index" (PI) of Ki 67 or any of proliferation antigens is number of antigen positive nuclei divided by the total number of nuclei in sampled microscopic areas of the tumour. Ki 67 LI varies widely between tumours and among different areas in one tumour. (16) The slides are screened to find areas with most and least stained nuclei. In each case, one thousand tumour cell nuclei from 8-12 randomly selected high -power fields (40x) in histological viable areas are counted for the positive and negative nuclei. Endothelial cells, inflammatory cells are excluded. Ki 67 labelling index (Ki 67 LI: percentage of Ki 67 positive nuclei/1000 tumour cells) is calculated for each neoplasm using a microscope. For each group of tumours, the Ki 67 LI, mean and standard deviation (SD) was statistically determined, and the distribution of $\mathrm{Ki} 67 \mathrm{LI}$ values is observed. (3) 


\section{Statistical Analysis}

The observations in our study are analyzed using Statistic Package Programmed for Social Science (SPSS software version 11.5) and applying the ANOVA test. Distribution \& frequency were obtained. The mean Mitotic Index +/Standard Deviation, the mean Ki $67 \mathrm{LI}+/$ - Standard Deviation values are statistically calculated using Microsoft Excel computer software. The observation between the morphological grading according to WHO 2007 classification, Mitotic Labelling Index and Ki 67 LI were analyzed, Ki 67 LI\% for each neoplasm and, the Ki 67 LI mean and standard deviation (SD) \% for each group of neoplasm were statistically determined and the distributions of Ki $67 \mathrm{LI}$ values are analyzed.

\section{RESULTS}

A total of 50 CNS tumours were studied. 3 metastatic CNS lesions were also incorporated. Non-neoplastic lesions were excluded and only neoplastic cases were selected which were sent in $10 \%$ buffered formalin. Antigen retrieval was achieved on all sections adequately. Out of 50 cases in our study $88 \%$ of the tumours were located intracranial and $12 \%$ were located in the spinal cord.

In our study the CNS tumours were more frequent in males than in females. Males comprised of $52 \%$ and females $48 \%$ of all the cases. In our study group adults comprised of 80 percent and $<19$ years age group (children and teenagers) comprised of 20 percent of all CNS tumours. Our study shows a maximum distribution of CNS tumours in Temporal lobe $18 \%$ followed by Frontal lobe-14\%, Cerebral meninges $-12 \%$, Posterior fossa and IV ventricle-8\% each, Parietal lobe- $8 \%$, Occipital and Fronto-parietal lobe- $6 \%$ each, Intra-Dural- $4 \%$, cerebellopontine angle $-2 \%$, Suprasellar region $-2 \%$, Craniopharyngeal duct $-2 \%$, thalamus- $2 \%$ and Extra cranial [spinal cord and spinal meninges] together- 4\%. There were no cases seen in the pineal gland or the cauda equine. In our study of sample population, age group 40-49 shows a higher frequency comprising of $15 \%$ to be affected by CNS tumours followed by 10-19, 30-39 and 60-69 groups comprising of $8 \%, 8 \%$ and $6 \%$ respectively. Gliomas was the most frequent $28(56 \%)$ followed by the non-glial tumour Meningioma 13 (26\%), Nerve sheath tumours $3(6 \%)$, Metastatic tumour 3 (6\%) and Pituitary adenomas 1 (2\%).

Medulloblastoma comprised 1 (2\%), and Craniopharyngioma 1 (2\%). Gliomas affected the 40-49 yrs age group more frequently with lower incidences at extremes of age but were the most common tumour in all age groups. Meningiomas and pituitary adenomas were seen to affect all age groups after 20 years. Medulloblastoma was seen in less than 20 yrs. Nerve sheath tumours were seen to affect all age beyond 30 yrs., 1 craniopharyngioma was seen in the 2nd decade, and 3 metastatic tumours out of which 2 beyond 70 yrs. and one in $5^{\text {th }}$ decade.

Our study showed gliomas occurring in males frequently while meningiomas were seen more frequently in females. 1 of 1 Pituitary adenoma and medulloblastoma respectively occurred in female, aggressive metastatic tumour was more in female and nerve sheath tumour was seen with female predominance. 1 of 1 craniopharyngioma occurred in male.

Tumours graded according to WHO 2017 classification fell most commonly in grade I comprising of $42 \%$ followed by grade II - $24 \%$, grade IV $-22 \%$ and grade III $-12 \%$ in our study group. In the entire spectrum of CNS tumours in our study a maximum of WHO Grade I tumours was comprised of diffuse fibrillary astrocytoma (Fig. 2), craniopharyngioma, meningiomas, pituitary adenomas and the Nerve sheath tumours. Grade II is comprised of gliomas, Grade III of oligoastrocytoma and anaplastic astrocytoma and Grade IV of glioblastoma multiforme (Fig. 3), metastatic tumours \& medulloblastoma. Our study shows most WHO grade I tumours in males as compared to females. This is due to meningiomas and low grade gliomas occurring frequently in females. Grade II frequently occur in males. 28 Gliomas in our study shows the following range of Ki $67 \mathrm{LI} \%$ for different WHO Grades - Grade I: 2.3-6.0, Grade II: 2.8-12, and Grade III: 4-10.8 \& Grade IV: 15-26.4 with mean Ki 67 LI ( \pm SD) values for grade I, II, III and IV gliomas areas follows: $\{2.62(+/-0.6)\}$, $4.76(+/-2.83) \%, 7.48(+/-2.53) \%$ and 18.44 (3.97) \% respectively.

\begin{tabular}{|c|c|c|c|}
\hline $\begin{array}{l}\text { WHO } \\
\text { Grade }\end{array}$ & Type of Tumour & $\begin{array}{l}\text { Mean MI } \\
(+/-S D)\end{array}$ & $\begin{array}{c}\text { Mean Ki } 67 \text { LI } \\
(+/-S D)\end{array}$ \\
\hline \multirow{5}{*}{$\begin{array}{l}\text { Grade I } \\
(21)\end{array}$} & Meningioma (10) & $0.99(+/-1.07)$ & $3.85(+/-1.97)$ \\
\hline & & $9(+/-0.82)$ & $3.0(+/-2.97)$ \\
\hline & Pituitary & 0.2 & 0.6 \\
\hline & & 0.3 & 1.1 \\
\hline & & $0.92(+/-0$. & $2.62(+/-0.60)$ \\
\hline \multirow{6}{*}{$\begin{array}{l}\text { Grade II } \\
(12)\end{array}$} & Gemisto & 1.8 & 12 \\
\hline & Pleomorp & $2.2(+/-0.28)$ & $4.3(+/-0.99)$ \\
\hline & & $0.9++/-0$. & $3.3(+/-0.71)$ \\
\hline & & $1.65(+/-1.3)$ & $3.9(+/-0.88)$ \\
\hline & Angi & 0.2 & \\
\hline & Atypical Mucinous meningioma (2) & $0.3(+/-1.4)$ & $6.12(+/-1.06)$ \\
\hline \multirow[t]{2}{*}{$\begin{array}{l}\text { Grade III } \\
\text { (6) }\end{array}$} & Anaplastic Astrocytoma (5) & $2.54(+/-0.94)$ & $6.82(+/-2.17)$ \\
\hline & Anaplastic Oligoastrocy & 1.6 & 10.8 \\
\hline \multirow{3}{*}{$\begin{array}{l}\text { Grade IV } \\
\text { (11) }\end{array}$} & Glioblastoma Multiforme (7) & $6.16(+/-0.72)$ & $18.44(+/-3.97)$ \\
\hline & & & \\
\hline & Metastatic tumour (3) & $19.33(+/-10.07)$ & $36.4(+/-22.16)$ \\
\hline & hle 1. Different WHO Grad & NS Tumours wi & $\begin{array}{l}\text { Their } \\
\text { SD) }\end{array}$ \\
\hline
\end{tabular}

\begin{tabular}{|ccc|}
\hline WHO Grade & Mean MI (+/-SD)\% & Mean Ki 67 LI (+/- SD)\% \\
Grade I & $0.92(+/-0.80)$ & $3.28(+/-2.26)$ \\
Range & $0.2-3.2$ & $0.6-9$ \\
Grade II & $1.38(+/-0.70)$ & $4.98(+/-3.12)$ \\
Range & $0.2-2.4$ & $2-12$ \\
Grade III & $2.38(+/-0.92)$ & $7.48(+/-2.53)$ \\
Range & $3.8-1.4$ & $4-10.8$ \\
Grade IV & $7.00(+/-2.10)$ & $24.42(+/-13.39)$ \\
Range & $5-12$ & $15-61$ \\
\multicolumn{2}{r}{ Table 2. Mean MI and Mean Ki 67 LI (+/-SD)\% } \\
& of WHO Grade I to IV CNS Tumours \\
\hline
\end{tabular}

Our study on 13 meningiomas shows the following range of Ki 67 LI \% for different WHO Grades- Grade I 1.3-8 Grade II 2-7.5 with mean $\mathrm{Ki} 67 \mathrm{Li}$ of $3.85(+/-1.97)$ and $5.17(+/-$ 2.84) respectively. We had no cases of Grade III meningioma in our study Ki 67 LI \% of 3 Nerve Sheath tumours is in the range of $1.2-6.4 \%$ in our study. Ki 67 LI \% of 1 metastatic follicular carcinoma to brain, 1 metastatic renal cell carcinoma and 1 metastatic papillary adenocarcinoma is in range of 18-61 with mean Ki LI of 36.4 (+/-22.16).

Overall our study showed the following range of Ki $67 \mathrm{LI}$ $\%$ for different WHO Grade CNS Tumours- Grade I 0.6-8, Grade 2-12, Grade III-4-10.8 \& Grade IV -15-61. The mean Ki$67 \mathrm{LI}+$ /- SD of various WHO grades of CNS tumours in our study is as follows (Table 2)- 
- $\quad$ Grade I- $3.09(+/-1.89) \%$

- $\quad$ Grade II- $4.86(+/-2.70) \%$

- $\quad$ Grade III- $7.48(+/-2.53) \%$

- $\quad$ Grade IV- 23.48 (+/-13.29) \%

\section{DISCUSSION}

To Know the malignant potential of brain tumours Growth rate is an essential feature. To assess tumour proliferation, histopathological grading by using WHO 2007 classification of CNS tumour is "Gold Standard", although it is known to be filled with difficulties because of the problems existing in mitotic labelling.(17) The current study was done to correlate morphological grading (according to WHO 2007 classification) with Ki 67 labelling index as a marker for proliferative activity of brain tumours. In our study cranial vs spinal distribution is $88 \%$ and $12 \%$ respectively, spinal distribution matches the $10-20 \%$ range mentioned in literature. The distribution in children $(20 \%)$ is as expected for the population.(18)

Morphological tumour types were encountered in the expected distribution range. Gliomas- 28 (56\%), Meningioma-13 (26\%), Pituitary Adenoma -1 (2\%), Nerve sheath tumour $-3(6 \%)$, metastatic tumours $-3(6 \%)$, Medulloblastoma- 1 (2\%) and Craniopharyngioma-1 (2\%), is comparable to epidemiological study of 34,140 cases over 60 years by Chen et. al., (19) except Pituitary Adenoma which is in accordance to $6-10 \%$. (20) This difference between Chen and our study could be due to sample size. Males outnumber females in accordance with IARC 2004. Meningioma occurred more frequently in females. Meningiomas exceeds Gliomas in spinal cord as in literature.

Kayaseleuk et al. (2002) study which shows the mean Ki $67 \mathrm{LI}+/$ - SD as follows: Grade I-1.58+/-3.74, GradeII-2.41+/4.08, Grade III $-25.96+/-19.1 \&$ Grade IV $-31.44+/-22.25$. The mean Ki $67 \mathrm{LI}+/$ - SD of the different WHO Grades in our study is as follows: Grade I-3.28 $(+/-2.26) \%$, GradeII-4.98 $(+/-3.12) \%$, Grade III $-7.48(+/-2.53) \%$ \& Grade IV-24.42 $(+/-13.39) \%$.

When our results were compared with Kayaseleuk et al. the mean Ki 67 LI of Grade I, II and IV are comparable. Variation in Grade III CNS tumours is due to presence of mainly anaplastic astrocytomas only one anaplastic oligodendroglioma (Ki $67 \mathrm{LI}-10.8$ ) is present in our study. The mean Ki 67 LI +/- SD of Grade III and Grade IV are comparable to the above study and to Burger PC et al (1986) study of higher LI that was associated with histologically malignant neoplasms (metastatic carcinomas, anaplastic mixed glioma and some glioblastomas). (5) Ki 67 LI of 6 ependymomas are $2.62(+/-0.60) \%$ ranges between 2.3-6.0. Karamitopoulou et al in their study shown Ki 67 LI for ependymoma $-2.63+/-2.58 .(21)$

The Clinical Value of Ki-67/MIB-1 Labelling Index in Human Astrocytomas by Johannessen AL et al's (2006) study highlights the main findings and conclusions from the 16 reviewed articles. All studies showed an increase in Ki/MIB-1 LI with the increasing grade of malignancy. Mean values $( \pm$ SD) for grade II, III and IV tumours for whole material 3.0 ( \pm 2.1$), 11.8( \pm 3.4)$ and $15.8( \pm 7.4)$, respectively. (22) In our study the mean Ki 67 LI ( \pm SD) values for Grade II, III and IV
Glioma is as follows - $4.76(+/-2.83), 7.48(+/-2.53)$ and $18.44(+/-3.97)$, respectively. Hence our study has been comparable to the many reviewed articles by Johannessen et al.

Cut offs point for prognostic values have been different in different studies on astrocytomas but a cut off set at Ki 67/MIB $1 \mathrm{LI}</=2.0$ was associated with longer survival according to study by McKeever et al and a cut off of $>8 \%$ shown in 2 studies by De et al and Schiffer et al was associated with significantly poor survival and Ki 67/MIB-1 LI $>20 \%$ in multivariate analysis done by Reavey-Cantwell's study shown to have 2.2 times the risk of death compared with patients with LI $<20 \%$. It is evident that majority of reviewed studies show prognostic significance of Ki 67/MIB 1 LI both associated with survival and recurrence, with proposed cut off values which varies greatly from $1.5 \%$ to $15.3 \%$. It is not easy to compare results between different reports and extract a clear conclusion. So, a Ki 67/MIB 1 LI $>10.0 \%$ has been a reasonable guideline value to indicate an astrocytic tumour with increased malignant potential. Our study shows a Ki 67 LI exceeding the cut offs as follows$>10 \%$ in 1 out of 9 Astrocytoma Grade II and all 7 cases of GBM.

It is concluded by above study that Ki $67 \mathrm{LI}$ serves as an important clinical marker in human astrocytomas. It should be used judiciously and in combination with various modalities like radiography, clinical status, duration of symptoms and established histopathological features of anaplasia. Ki $67 \mathrm{LI}$ is of particular importance in cases with low grade histology when other factors indicate a more malignant neoplasm.

Meningioma is classified as typical, atypical meningiomas (at least 4 mitoses per $10 \mathrm{HPF}$.) and malignant meningiomas (at least 20 mitoses per $10 \mathrm{HPF}$ ).(23) In our study 5 meningothelial meningiomas with 1 fibroblastic, 4 psammomatous meningioma (total 10) WHO Grade I types with mean $\mathrm{Ki} \mathrm{LI}+/-\mathrm{SD}$ of 4.31 (+/-2.78), range: 1-9.9 which corresponds to the range in the study by Pinar (range 0\%19.9\%), 1 atypical angiomatous meningioma and 2 atypical mucinous meningioma WHO Grade II shows Ki LI of -3.4 (1.57) correlating with Pinar's study.(24)

In our study of 3 Peripheral nerve sheath tumours, 2 are $\mathrm{CP}$ angle Schwannomas with 1 intradural extra medullary Schwannoma. The mean Ki $67 \mathrm{LI}+/-S D$ is 2.97 (+/-2.97) \%, range- 1.2 to $6.4 \%$. 2 Schwannomas have Ki $67<4.0 \%$ except one schwannoma with $6.4 \%$, however no BPNST's showed Ki $67>7 \%$ or a high mitotic count as for a MPNST. A count of $>$ $4.0 \%$ is usually seen in a cellular schwannoma. (25)

Karamitopoulou et al study on 136 CNS tumours mean Ki 67 LI (+/-SD) for medulloblastoma is 18.77 $\pm 9.65 .(21)$ Kayaseleuk et al. (2002) detected highest Ki- 67 LI values in medulloblastoma, malignant meningioma, primitive neuroectodermal tumour (PNET) and glioblastoma (GBM) groups, the mean $\mathrm{Ki}-67 \mathrm{LI}+/-\mathrm{SD} \%$ was $31.44(+/-22.55)$. In our study mean Ki $67 \mathrm{LI}$ (+/-SD) \% for 1 medulloblastoma is 30.3 , correlates with Kayaseleuk et al values. 3 Metastatic tumours - 1 metastatic follicular carcinoma to brain and 1 metastatic renal cell carcinoma to brain $-77 \%$ and 1 metastatic papillary adenocarcinoma - with mean Ki 67 LI (+/-SD) \% - 36 (+/-22.16) \% have shown the highest Ki $67 \%$ in our study. Quifiones-H et al have concluded that their 
findings indicated that malignant CNS tumours always exhibited high Ki 67 LI value.(4)

A long-term follow-up of subjects in this study will be required to substantiate the perceived value of Ki 67 LI of these cases as no reliable and reproducible established cut offs are available.

\section{CONCLUSIONS}

Ki 67 LI serves as an essential clinical prognostic proliferation marker of particular importance in cases with lower grade histology of Grade II \& Grade III astrocytomas, Grade II \& Grade III oligodendrogliomas. Ki 67 LI is important in determining benign, atypical and malignant meningiomas, non-invasive and invasive pituitary adenomas.

\section{REFERENCES}

[1] Sabiston DC, Townsend CM, Beauchamp RD. Sabiston textbook of surgery the biologic basis of modern surgical practice. 14th edn. W.B. Sunder's Company 1991.

[2] Arshad H, Ahmad Z, Hasan SH. Gliomas: correlation of histologic grade, Ki67 and p53 expression with patient survival. Asian Pac J Cancer Prev 2010;11(6):1637-40.

[3] Kayaselcuk F, Zorludemir S, Gumurduhu D, et al. PCNA and Ki-67 in central nervous system tumours: correlation with the histological type and grade. J Neurooncol 2002;57(2):115-21.

[4] Quiñones-Hinojosa A, Sanai N, Smith JS, et al. Techniques to assess the proliferative potential of brain tumours. J Neurooncol 2005;74(1):19-30.

[5] Burger PC, Shibata T, Kleihues P. The use of the monoclonal antibody Ki-67 in the identification of proliferating cells: application to surgical neuropathology. Am J Surg Pathol 1986;10(9):611-7.

[6] Montine TJ, Vandersteenhoven JJ, Aguzzi A, et al. Prognostic significance of Ki-67 proliferation index in supratentorial fibrillary astrocytic neoplasm. Neurosurgery 1994;34(4):674-9.

[7] McKeever PE, Ross DA, Strawderman MS, et al. A comparison of the predictive power for survival in Gliomas provided by MIB-1, bromodeoxyuridine and proliferating cell nuclear antigen with histopathologic and clinical parameters. J Neuropathol Exp Neurol 1997;56(7):798-805.

[8] Sallinen PK, Haapasalo HK, Visakorpi T, et al. Prognostication of Astrocytoma patient survival by Ki-67 (MIB-1), PCNA, and S-phase fraction using archival paraffin -embedded samples. J Pathol 1994;174(4):27582.
[9] Louis DN, Ohgaki H, Wiestler OD, et al. World Health Organization histological classification of tumours of the central nervous system. International Agency for Research on Cancer, France 2016.

[10] Kayaselçuk F, Zorludemir S, Gümürdülü D, et al. PCNA and Ki-67 in central nervous system tumours: correlation with the histological type and grade. J Neurooncol 2002;57(2):115-21.

[11] Russell DS, Rubinstein LJ. Pathology of tumours of the nervous system. The Journal of Pathology 1989;158(4):359.

[12] Chaloob MK, Ali HH, Qasim BJ, et al. Immunohistochemical expression of Ki-67, PCNA and CD34 in astrocytomas: a clinicopathological study. Oman Med J 2012;27(5):368-74.

[13] Gejman R, Swearingen B, Tessa E, et al. Role of Ki-67 proliferation index and p53 in predicting progression of pituitary adenomas. Hum Pathol 2008;39(5):758-66.

[14] Dako. Envision TM Flex Mini Kit, High Ph, (Link). Code K8023/EFG/NAN;2011.

[15] Thermoscientific. "Ki 67 (Clone SP6) (Rabbit Monoclonal Antibody), Invitro Diagnostic Data Sheet". Rev01081H; 2013.

[16] Jansen M, Yip S, Louis DN. Molecular pathology in adult Gliomas: diagnostic, prognostic, and predictive markers. Lancet Neurol 2010;9(7):717-26.

[17] de Wolde H, Pruim J, Mastik MF, et al. Proliferative activity in human brain tumours: comparison of histopathology and L -[1 -HC] tyrosine PET. J Nuc Med 1997;38(9):1369-74.

[18] Boyle P, Ferlay J. Cancer incidence and mortality in Europe, 2004. Ann Oncol 2005;16(3):481-8.

[19] Daumas-Duport C, Tucker ML, Kolles H, et al. Oligodendrogliomas. Part II: a new grading system based on morphological and imaging criteria. J Neurooncol 1997;34(1):61-78.

[20] Blevins LS, Verity DK, Allen G. Aggressive pituitary tumours. Oncology 1998;12(9):1307-12.

[21] Karamitopoulou E, Perentes E, Diamantis I, et al. Ki-67 immunoreactivity in human central nervous system tumours: a study with MIB 1 monoclonal antibody on archival material. Acta Neuropathol 1994;87(1):47-54.

[22] Johannessen AL, Torp SH. The clinical value of $\mathrm{Ki}$ 67/MIB-1 labelling index in human astrocytomas. Pathol Oncol Res 2006;12(3):143-7.

[23] Bruna J, Brell M, Ferrer I, et al. Ki-67 proliferative index predicts clinical outcome in patients with atypical or anaplastic meningioma. Neuropathology 2007;27(2):114-20.

[24] Kang SK, Chang SE, Choi JH, et al. A case of cellular schwannoma of the skin presenting as a large ulcerated tumour on the ankle. J Dermatol 2002;29(1):28-32.

[25] Kim DH, Suh YL, Shin DI, et al. p53 expression and Ki-67 labelling index in brain tumour with special reference to tumour and histologic grade. Korean J Pathol 1998;32(2):81-7. 